\title{
Análise de Sensor SPR na Configuração SCPE Baseado em Camada Dupla de Grafeno em THz
}

\author{
Yago G. da Conceição, André F. S. Cruz, Wendria C. da Silva, Andrey V. Pires, Patrik C. Lopes, Paulo R. \\ Amaral e Karlo Q. da Costa
}

\begin{abstract}
Resumo-Este trabalho apresenta a análise paramétrica do sensor SPR na configuração SPCE baseado em duas camadas de grafeno. Realiza-se a análise pelo Método dos Elementos Finitos, calculando os campos próximos e distantes na estrutura do sensor. $O$ modelo é formado por uma estrutura multicamadas: Ar/ Grafeno/ $\mathrm{SiO}_{2} /$ Grafeno/ BK7. Na região do Ar localiza-se um dipolo hertziano infinitesimal operando $\operatorname{com} \lambda \simeq 250 \mu \mathrm{m}$ simulando os analitos a serem detectados na resposta de saída do sensor. Verifica-se o acoplamento do ondas TM na região do prisma analisando a melhoria na diretividade e sensibilidade do sensor.
\end{abstract}

\section{Palavras-Chave-Grafeno, SPR, SPCE.}

Abstract-This work presents the parametric analysis of the SPR sensor in the SPCE configuration based on two layers of graphene. The analysis is performed by the Finite Element Method, calculating the near and far fields in the sensor structure. The model is formed by a multilayer structure: Air/ Graphene/ $\mathrm{SiO}_{2} /$ Graphene/ $\mathrm{BK} 7$. In the region of $\mathrm{Ar}$ there is an infinitesimal hertzian dipole operating with $\lambda \simeq 250 \mu \mathrm{m}$ simulating the analytes to be detected in the sensor's output response. The TM waves are coupled in the prism region, analyzing the improvement in the directivity and sensitivity of the sensor.

Keywords-Graphene, SPR, SPCE.

\section{INTRODUÇÃO}

O grafeno é formado por átomos de carbono que se arranjam em forma de hexágonos e tem estrutura bidimensional obtido experimentalmente [1] que juntamente com fósforo negro, Nitreto de boro hexagonal (hBN) e Dissulfeto de Molibdênio $\left(\mathrm{MoS}_{2}\right)$ [2] formam as estruturas bidimensionais, tendo-se como ponto de vista a física do estado sólido.

Desde seu isolamento em 2004 [3], o grafeno tem se tornado um material muito promissor devido suas propriedades eletromagnéticas, eletromecânicas e sua excelente condutividade elétrica [4], podendo ser obtido (por ser uma forma alotrópica do carbono) através do grafite. Estruturalmente o grafite pode ser entendido como uma pilha de camadas individuais de átomos de carbono. De fato, $1 \mathrm{~mm}$ de grafite possui cerca de 3 milhões de camadas individuais separadas umas das outras

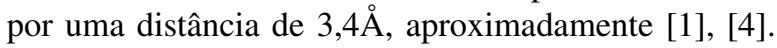

O sensor SPR (Surface Plasmon Resonance) trata-se de um dispositivo fotônico capaz de detectar alterações no índice de refração eletromagnético na sua superfície, podendo essas

Y. G. Conceição, A. F. S. Cruz, W. C. Silva, Andrey V. Pires, P. C. Lopes, P. R. Amaral, K. Q. Costa, Programa de Pós-Graduação em Engenharia Elétrica, Instituto de Tecnologia, Universidade Federal do Pará, Belém-PA, Brasil, E-mails: yagogc.ee@gmail.com, andcruz@ufpa.br, wendria.cunha@gmail.com, andrey.viana@live.com, patrick14lopes@gmail.com, pauloamaral2708@gmail.com e karlo@ufpa.br alterações estarem relacionadas a interações intermoleculares ou a detecção de analitos imobilizados [4], a partir da interação entre as amostras analisadas e o campo evanescente gerado pela onda SPP (Surface Plasmon Polariton), que se propagam na interface metal-dielétrico [5].

SPPs foram referenciadas pela primeira vez no início do século passado, mas somente no início da década de 1980, dispositivos baseados em SPP começaram a ser aplicados em sensores ópticos [5] com aplicações em detecção de gás e biossensores, caracterizando e quantificando as interações biomoleculares e diagnósticos médicos para monitoramento viral [4].

Um sensor SPR convencional na configuração SPCE (Surface Plasmon Coupled Emission) é formado por uma estrutura multicamadas acoplada a um canal de microfluídica por onde passam analitos, que podem ser imobilizados sobre a estrutura do sensor, que por sua vez é excitado por uma fonte externa [4], [5].

No espectro óptico, a operação do sensor SPCE é baseada no fenômeno da ressonância plasmônica que ocorre em metais nobres submetidos a altas frequências. Na faixa de Terahertz, os metais são altamente refletivos, o que impede o funcionamento deste dispositivo nessa frequência. Para contornar esse problema, o grafeno tem sido proposto por apresentar baixas perdas em Terahertz [4], [6], além de suportar ondas plasmônicas. Trabalhar com esse dispositivo na faixa $\mathrm{THz}$ possibilita aumentar suas dimensões [4], [5] o que reduz os custos de fabricação do sensor [4].

Este trabalho apresenta uma análise numérica de um sensor SPR na configuração SPCE baseado em dupla camada grafeno na faixa THz. Foram realizadas simulações no ambiente COMSOL Multiphysics ${ }^{\circledR}$, que tem por base o Método dos Elementos Finitos para resolver os campos próximos e distantes. Análises paramétricas foram realizadas no near field e far field, verificando a influência na resposta do sensor quanto a orientação e altura do dipolo hertziano que modela o comportamento da partícula alvo. O modelo na faixa de $\mathrm{THz}$ apresentado aqui já foi validado em outros trabalhos [4], [6], assim, o foco aqui será uma análise paramétrica mais detalhada e aprofundada.

\section{Modelagem NumÉRICA DO SENSOR}

A estrutura trabalhada (Fig. 1) é composta por multicamadas (Ar/ Grafeno/ $\mathrm{SiO}_{2} /$ Grafeno/ Prisma BK7) e é excitada por uma fonte externa que opera com comprimento de onda $\lambda$ na faixa $\mathrm{THz}$. 


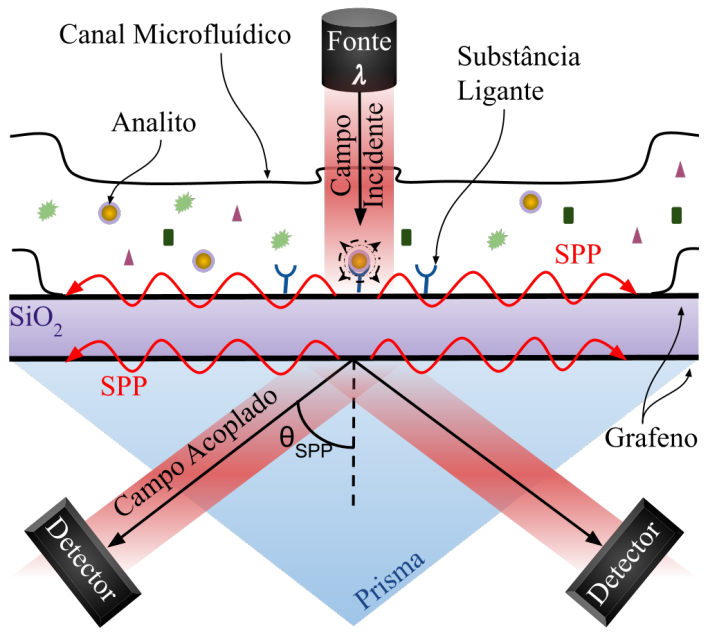

Fig. 1. Modelo funcional do sensor SPR na configuração SPCE acoplado a um canal microfluídico.

Os analitos são imobilizados sobre a superfície do sensor por meio de uma substância ligante que tem afinidade química com a partícula mas que seja eletricamente inerte [4], sendo então, excitada por uma fonte laser que opera na faixa terahertz com $\lambda \simeq 250 \mu \mathrm{m}$.

Quando excitadas as nanopartículas re-irradiam ondas no mesmo comprimento de onda que excitam ondas SPP nas interfaces com camadas de grafeno acoplando ondas altamente polarizadas em modo transversal magnético na região do prisma. A resposta eletromagnética do sensor aparece como um cone na região do prisma [4], onde o ângulo do abertura $\theta_{S P P}$ está em função do índice de refração efetivo do canal microfluídico. Essa resposta é lida por detectores na parte inferior do dispositivo.

A modelagem numérica do sensor SPR foi realizada no ambiente de simulação 3D do software COMSOL Multiphysics ${ }^{\circledR}$ com base na geometria apresentada na Fig. 2, onde a camada referente ao canal microfluídico está apresentada como a camada de Ar e a partícula alvo e seu processo de re-irradiação (inicialmente excitada por uma fonte externa) é equivalentemente relacionada com a emissão de um dipolo hertziano equivalente [4]-[6] com momento de corrente normalizado operando com comprimento de onda $\lambda$.

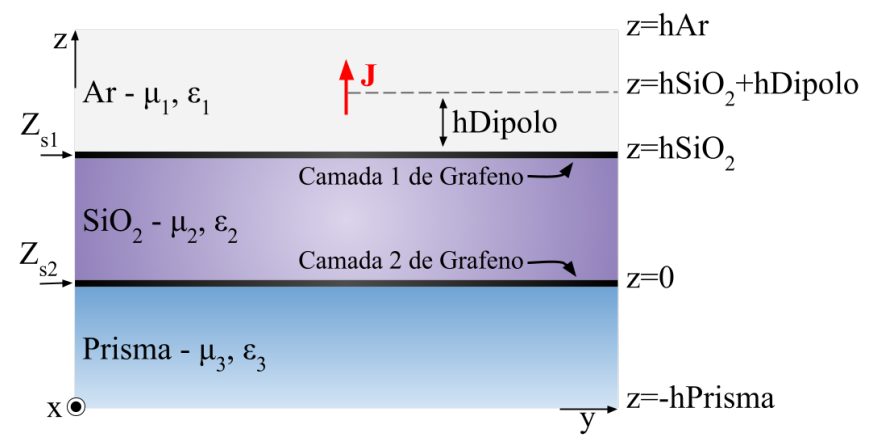

Fig. 2. Modelo equivalente do sensor multicamadas na configuração SPCE.

A altura do dipolo em relação a primeira camada do dispositivo representa o tamanho da substância ligante que fixa o analito sobre o sensor [5]. Foram realizadas análises de campo elétrico e magnético variando a altura do dipolo hertziano (Fig. 3a) dada por $h_{\text {Dipolo }}=h(\lambda / 632.8)$ sendo $h$ variado em $h=20 \mu \mathrm{m}, h=100 \mu \mathrm{m}$ e $h=200 \mu \mathrm{m}$ para cada uma das orientações do dipolo $\theta^{\prime}=0^{\circ}, \theta^{\prime}=45^{\circ}$ e $\theta^{\prime}=90^{\circ}$ com $\phi^{\prime}=0^{\circ}$. Essa análise serve para verificar a influência do separador químico bem como a fidelidade do modelo com dipolo representando a partícula alvo e seu processo de reirradiação.

O domínio espacial de simulação foi definido por uma esfera de raio $4 \lambda$ dividida em três camadas esféricas. A (Fig. 3b) mostra a malha discretizada do problema.

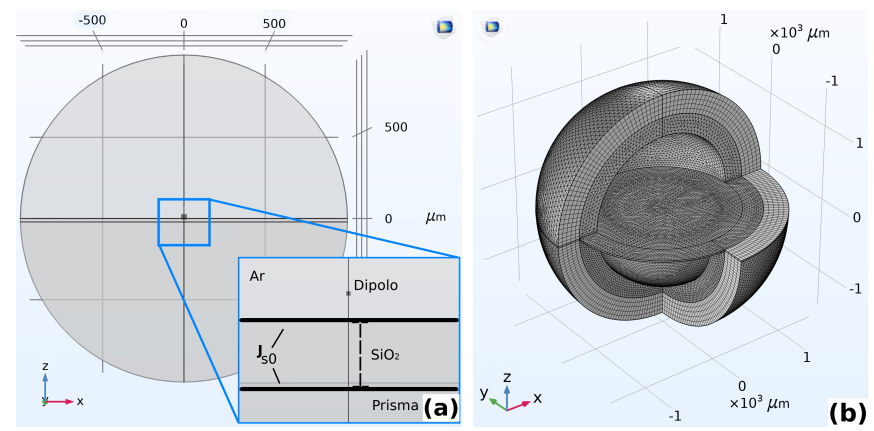

Fig. 3. Visão do dispositivo modelado no software: (a) detalhe da estrutura e identificação das camadas; (b) estrutura com malha discretizada.

A malha de discretização é formada em três definições. Na camada mais externa onde está definida a PML (Perfect Matched Layer - Fig. 4a), foi setado a definição de distribuição (Distribution) em modo Swept onde foi configurado para 6 elementos, discretizando a PML em 6 camadas.

Para a segunda camada (Fig. 4b), Far-Field Domain, foi escolhido a malha do tipo Tetraédrico Livre (Free Tetrahedral) com tamanho dos elementos predefinidos como Extra fine. No restante da estrutura, onde de fato está o sensor na configuração SPCE, também foi escolhido malha do tipo Tetraédrico Livre, mas com seu refinamento de malha sendo configurado para elementos Extremely fine.

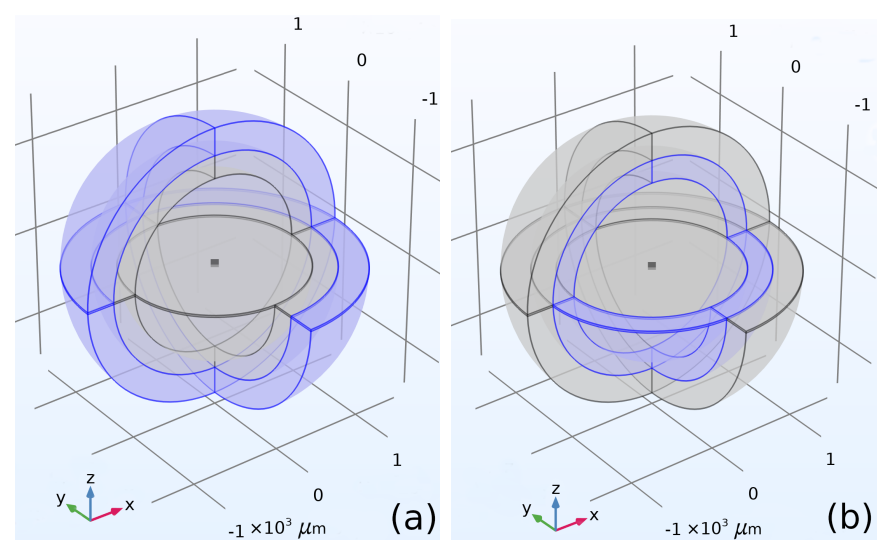

Fig. 4. Identificação das camadas: (a) camada absorvente (PML - Perfect Matched Layer); (b) Far-Field Domain.

A malha completa consiste em 219.595 elementos de domínio, 28.162 elementos de limite e 1.307 elementos de borda. 
Apenas uma simulação totalizou $11 \mathrm{~h} 6 \mathrm{~min}$ e 30 s em um computador de 64GB de memória RAM com processador Intel(R) Xenon(R) CPU E5-1650 3.50GHz de 12 núcleos.

O dipolo é modelado pela função Electric Point Dipole com especificação de Magnitude and direction sendo o Electric current dipole moment [7] dado pela Equação 1, sendo $\mathbf{n}_{p}=$ $1 \mathbf{a}_{x}+1 \mathbf{a}_{y}+1 \mathbf{a}_{z}$, com a componente $y$ zerada, uma vez que a orientação será analisada no plano $x z$.

$$
\mathbf{P}=p \mathbf{n}_{p} \quad A . m
$$

Para verificar a resposta eletromagnética do sensor à condição sem amostragem, o canal microfluídico foi modelado como uma camada de ar $\left(\varepsilon_{r 1}\right)$ enquanto a camada de $\mathrm{SiO}_{2}$ $\left(\varepsilon_{r 2}\right)$ foi definida com uma espessura de $\mathrm{hSiO}_{2}$ e posicionada entre duas camadas de grafeno. Na última camada foi usado o prisma óptico BK7 $\left(\varepsilon_{r 3}\right)$.

A Tabela I apresenta a relação dos parâmetros inseridos no COMSOL Multiphysics ${ }^{\circledR}$ para a modelagem da estrutura.

TABELA I

ParÂMETRos Para modelagem Da estrutura. [4]

\begin{tabular}{|c|c|c|}
\hline Nome & Expressão & Valor \\
\hline lamb & c_const/1.2[THz] & 2.4983E-4 m \\
\hline a & $4.5 * \operatorname{lamb}$ & $0.0011242 \mathrm{~m}$ \\
\hline far & lamb/5 & 4.9965E-5 m \\
\hline pml & lamb/5 & 4.9965E-5 m \\
\hline hDipolo & $20 *(\operatorname{lamb} / 632.8)$ & $7.8959 \mathrm{E}-6 \mathrm{~m}$ \\
\hline hSiO2 & $50 *(\operatorname{lamb} / 632.8)$ & $1.974 \mathrm{E}-5 \mathrm{~m}$ \\
\hline er_Prisma & 2.34 & 2.34 \\
\hline er_SiO2 & 2.132 & 2.132 \\
\hline $\mathrm{T}$ & $300[\mathrm{~K}]$ & $300 \mathrm{~K}$ \\
\hline uc & $0.5[\mathrm{eV}]$ & $8.0109 \mathrm{E}-20 \mathrm{~J}$ \\
\hline umb & $10^{\wedge} 4\left[\mathrm{~cm}^{\wedge} 2 /\left(\mathrm{V}^{*} \mathrm{~s}\right)\right]$ & $1 \mathrm{~m} \wedge 2 /(\mathrm{V} \cdot \mathrm{s})$ \\
\hline $\mathrm{vf}$ & $10^{\wedge} 6[\mathrm{~m} / \mathrm{s}]$ & $1 \mathrm{E} 6 \mathrm{~m} / \mathrm{s}$ \\
\hline tau & $(\mathrm{uc} * \mathrm{umb}) /\left(\left(\mathrm{vf}^{\wedge} 2\right)^{*} \mathrm{e} \_\right.$const $)$ & $5 \mathrm{E}-13 \mathrm{~s}$ \\
\hline gamma & $\operatorname{tau}^{\wedge}(-1)$ & $2 \mathrm{E} 12 \mathrm{l} / \mathrm{s}$ \\
\hline
\end{tabular}

O grafeno é um material considerado aproximadamente bidimensional, tendo espessura de até uma única camada atômica [4]. Por essa razão, podemos modelar o grafeno como uma impedância de superfície $Z_{s}=1 / \sigma_{g}$ na interface entre as camadas $\mathrm{Ar} / \mathrm{SiO}_{2}$ e $\mathrm{SiO}_{2} /$ Prisma. No COMSOL Multiphysics ${ }^{\circledR}$, isso é feito usando a condição de contorno de densidade de corrente superficial (Surface Current Density - Equação 2) [7] nas interfaces situadas em $z=0$ (Interface 1) e $z=h \mathrm{SiO}_{2}$ (Interface 2).

$$
\mathbf{J}_{s 0}=\sigma_{g}\left(E_{x} \mathbf{a}_{x}+E_{y} \mathbf{a}_{y}\right) \quad A . m
$$

Onde $\sigma_{g}$ é a condutividade superficial do grafeno, e $\left(E_{x} \mathbf{a}_{x}+E_{y} \mathbf{a}_{y}\right)$ é o campo elétrico tangencial a interface (Condição de Impedância) [6]. Na equação anterior $\sigma_{g}$ pode ser dado pela Equação 3 dividida em duas partes. Uma descrevendo as transições intrabanda (transições dentro da banda de condução, ou valência, onde o momento não é conservado - Equação 4) e outra responsável pelas transições interbanda (transições verticais da valência para a banda de condução, caso em que existe conservação do momento - Equação 5).

$$
\sigma_{g}(\omega)=\sigma_{\text {intra }}(\omega)+\sigma_{\text {inter }}(\omega)
$$

Uma expressão analítica para a condutividade dinâmica do grafeno pode ser obtida no âmbito da teoria da resposta linear e é calculada por meio da fórmula de Kubo [4], [8].

$$
\sigma_{\text {intra }}(\omega)=\frac{\sigma_{0}}{\pi} \frac{4}{\hbar(\gamma+i \omega)}\left[\mu_{c}+2 k_{B} T \ln \left(1+e^{-\mu_{c} / k_{B} T}\right)\right]
$$

A Equação 4 caracteriza a parcela intrabanda de $\sigma_{g}(\omega)$, onde $\sigma_{0}=e^{2} /(4 \hbar), k_{B}$ é a constante de Boltzmann, $\gamma=\tau^{-1}$ é a taxa de relaxamento [9] e $\mu_{c}$ potencial químico da folha de grafeno, enquanto que para a parcela interbanda temos a Equação 5 [10].

$$
\sigma_{\text {inter }}(\omega) \simeq \frac{-i e^{2}}{4 \pi \hbar} \ln \left(\frac{2\left|\mu_{c}\right|-(\omega-\gamma) \hbar}{2\left|\mu_{c}\right|+(\omega-\gamma) \hbar}\right)
$$

No grafeno sabe-se que o tempo de amortecimento $\tau$ depende do nível de dopagem do material, seja estático (processo químico) ou dinâmico (aplicação de um campo polarizador externo) [6]. Para grafeno acima de um substrato $\mathrm{SiO}_{2}$, esse parâmetro fenomenológico pode ser aproximado pela Equação 6 [9]. Onde $\mu_{m b}$ é a mobilidade eletrônica no grafeno, $e$ a carga do elétron e $v_{F}$ a velocidade de Fermi.

$$
\tau=\frac{\mu_{c} \mu_{m b}}{v_{F}^{2} e}
$$

A Tabela II apresenta a relação de descrição dos parâmetros para a condutividade do grafeno dada pela Equação 3 [4].

TABELA II

PARÂMETROS UTILIZADOS PARA CALCULAR A CONDUTIVIDADE SUPERFICIAL DO GRAFENO. [6], [8]

\begin{tabular}{cccc}
\hline & Valor & Unidade & Descrição \\
\hline$e$ & $1.6022 \mathrm{E}-19$ & $C$ & Electron charge \\
$\hbar$ & $1.0546 \mathrm{E}-34$ & $\mathrm{~J} \cdot \mathrm{s} / \mathrm{rad}$ & R. Planck constant \\
$k_{B}$ & $1.3806 \mathrm{E}-23$ & $\mathrm{~J} / \mathrm{K}$ & Boltzmann constant \\
$T$ & 300 & $\mathrm{~K}$ & Temperature \\
$v_{F}$ & $1 \mathrm{E} 6$ & $\mathrm{~m} / \mathrm{s}$ & Fermi velocity \\
$\mu_{m b}$ & $1 \mathrm{E} 4$ & $\mathrm{~cm} / \mathrm{V} \cdot \mathrm{s}$ & Electronic mobility \\
$\sigma_{0}$ & $e^{2} / 4 \hbar$ & $\mathrm{S}$ & AC Conductivity \\
$\mu_{c}$ & 0.5 & $\mathrm{eV}$ & Chemical potential \\
\hline
\end{tabular}

\section{RESULTADOS}

Modelada a estrutura no software e os parâmetros construtivos definidos, as simulações de campo próximo e distante foram realizadas na frequência de $1,2 \mathrm{THz}$. Potencial químico de $\mu_{c}=0.5 \mathrm{eV}$ foi aplicado igualmente às camadas de grafeno. A partir daí foi realizado análise do comportamento do modelo 
aos parâmetros de altura do dipolo elétrico (dipolo hertziano) e sua orientação. Os resultados são mostrados nas figuras seguintes.

Essa análise serve para verificar a influência do separador químico eletricamente inerte que liga o analito a superfície do sensor [4]. Foram realizadas análises de campo elétrico e magnético variando a altura do dipolo hertziano dada por $h_{\text {Dipolo }}=h(\lambda / 632.8)$ sendo $h$ variado em $20 \mu \mathrm{m}, 100 \mu \mathrm{m}$ e $200 \mu \mathrm{m}$ para cada uma das orientações do dipolo $\theta^{\prime}=0^{\circ}$ (Fig. 5), $\theta^{\prime}=45^{\circ}$ (Fig. 6) e $\theta^{\prime}=90^{\circ}$ (Fig. 7).
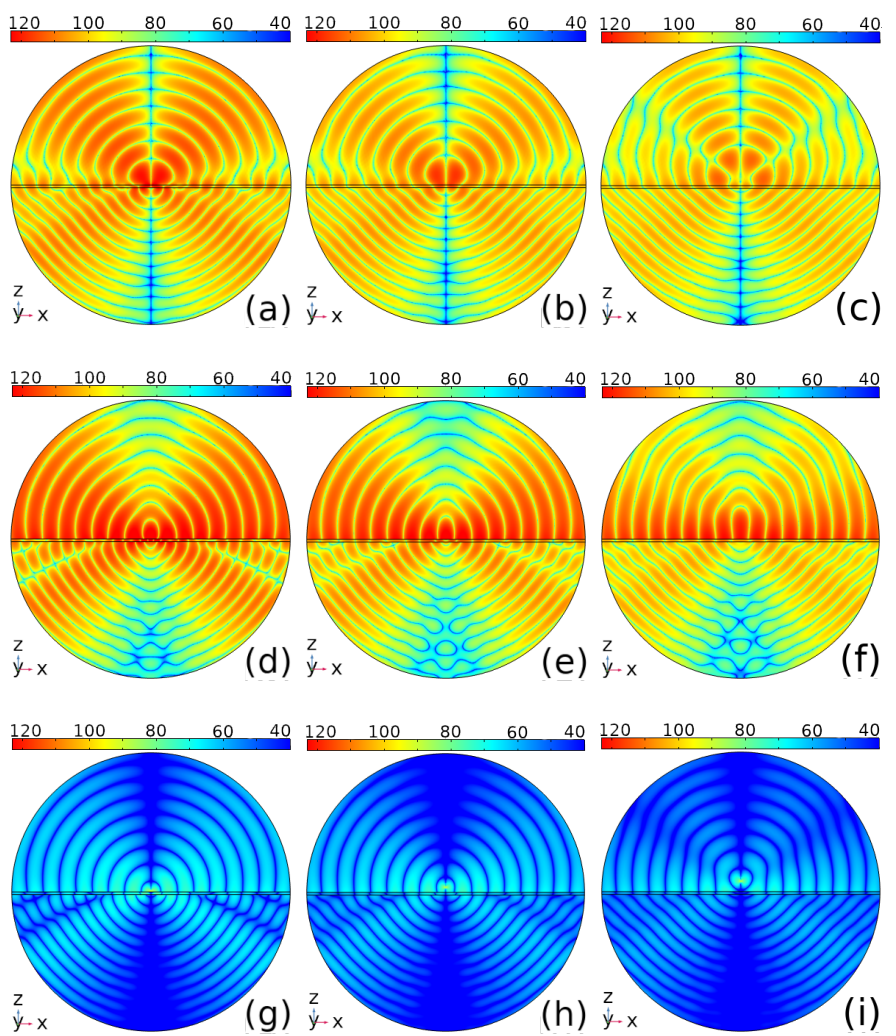

Fig. 5. Dipolo orientado na vertical (VED - $\theta^{\prime}=0^{\circ}$ ). $\mathbf{E}$ e $\mathbf{H}$ com $h_{\text {Dipolo }}=h(\lambda / 632.8) \cdot 20 \log \left(\left|\operatorname{Re}\left\{E_{x}\right\}\right|\right): \operatorname{com} h=$ (a) $20 \mu m$, (b) $100 \mu m$, (c) $200 \mu m .20 \log \left(\left|\operatorname{Re}\left\{E_{z}\right\}\right|\right): \operatorname{com} h=$ (d) $20 \mu m$, (e) $100 \mu m$ (f) $200 \mu m .20 \log \left(\left|\operatorname{Re}\left\{H_{y}\right\}\right|\right): \operatorname{com} h=(\mathrm{g}) 20 \mu \mathrm{m}$, (h) $100 \mu \mathrm{m}$, (i) $200 \mu m$
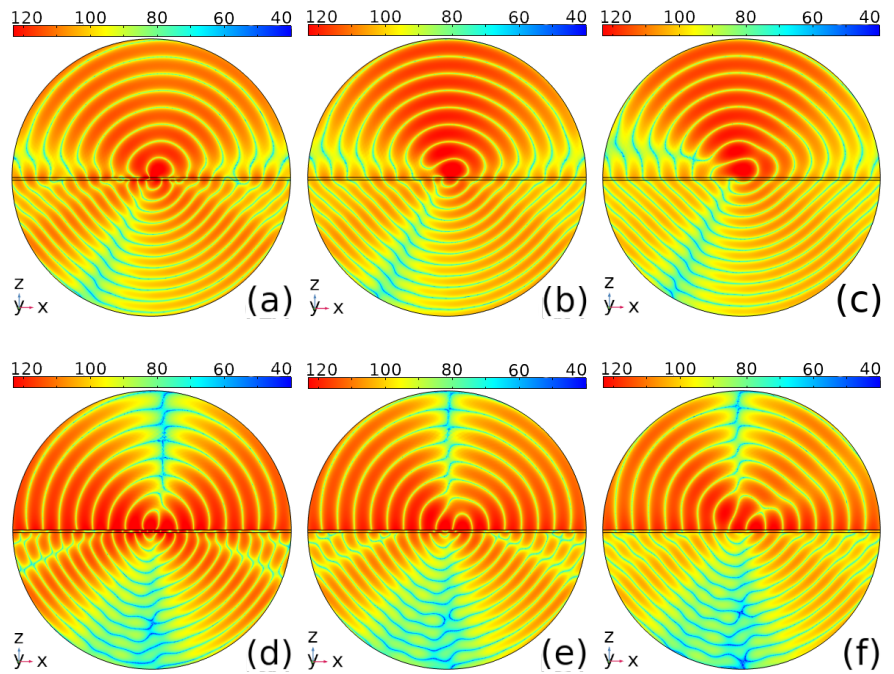
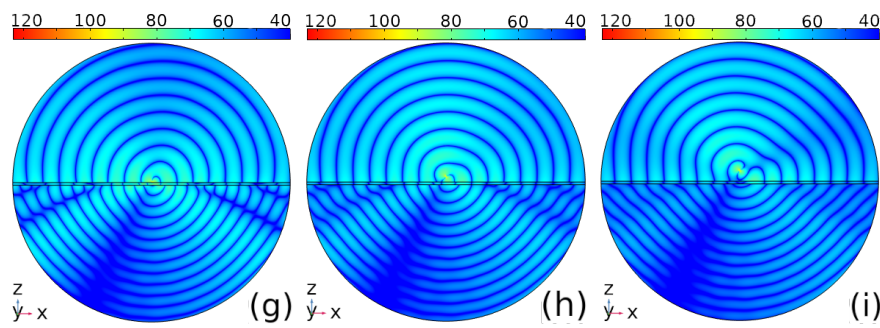

Fig. 6. Dipolo orientado na diagonal $\left(\theta^{\prime}=45^{\circ}\right)$. $\mathbf{E}$ e $\mathbf{H}$ com $h_{\text {Dipolo }}=$ $h(\lambda / 632.8) .20 \log \left(\left|\operatorname{Re}\left\{E_{x}\right\}\right|\right): \operatorname{com} h=$ (a) $20 \mu \mathrm{m}$, (b) $100 \mu \mathrm{m}$, (c) $200 \mu \mathrm{m} .20 \log \left(\left|\operatorname{Re}\left\{E_{z}\right\}\right|\right): \operatorname{com} h=$ (d) $20 \mu \mathrm{m}$, (e) $100 \mu \mathrm{m}$, (f) $200 \mu \mathrm{m}$. $20 \log \left(\left|\operatorname{Re}\left\{H_{y}\right\}\right|\right): \operatorname{com} h=(\mathrm{g}) 20 \mu \mathrm{m}$, (h) $100 \mu \mathrm{m}$, (i) $200 \mu \mathrm{m}$.
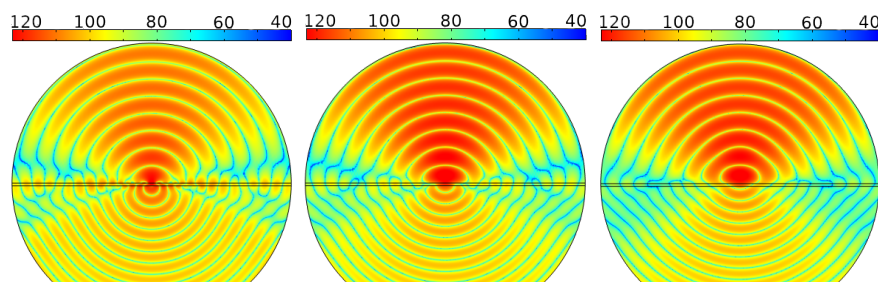

$z=x$

(a) $\underset{y-x}{z}$

(b) ${ }^{z}-x$

(c)
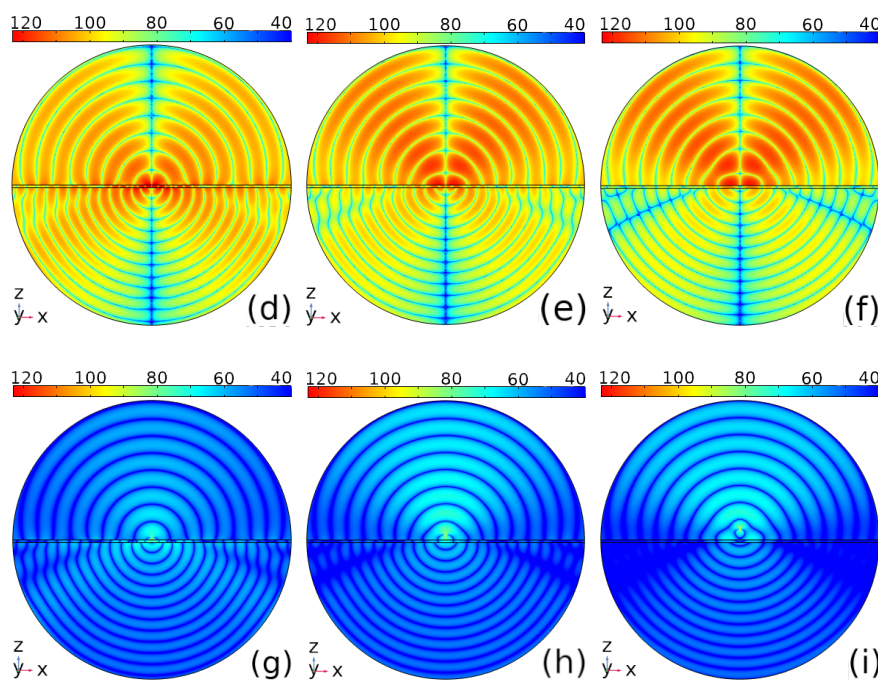

Fig. 7. Dipolo orientado na horizontal (HED - $\left.\theta^{\prime}=90^{\circ}\right)$. E e $\mathbf{H}$ com $h_{\text {Dipolo }}=h(\lambda / 632.8) \cdot 20 \log \left(\left|\operatorname{Re}\left\{E_{x}\right\}\right|\right): \operatorname{com} h=$ (a) $20 \mu m$, (b) $100 \mu \mathrm{m}$, (c) $200 \mu \mathrm{m} .20 \log \left(\left|\operatorname{Re}\left\{E_{z}\right\}\right|\right): \operatorname{com} h=$ (d) $20 \mu \mathrm{m}$, (e) $100 \mu \mathrm{m}$, (f) $200 \mu m .20 \log \left(\left|\operatorname{Re}\left\{H_{y}\right\}\right|\right): \operatorname{com} h=$ (g) $20 \mu m$, (h) $100 \mu m$, (i) $200 \mu \mathrm{m}$.

Os resultados obtidos mostram que a alteração do espaçamento entre o dipolo hertziano e a primeira camada de grafeno (que representa a altura da substância ligante) não introduz alterações significativas de campo próximo na região do prisma, mas altera a distribuição de campo no domínio caracterizado pelo ar (representando o canal microfluídico por onde a amostra contendo os analitos irá circular). Apesar disso, se a altura da substancia ligante for aumentada pode-se gerar uma excitação menos intensa de plasmons de superfície, motivo pelo qual é usado $20 \mathrm{~nm}$ nas referências [5], [11] e $h_{\text {Dipolo }}=20(\lambda / 632.8)$ neste trabalho.

Isso pode ficar mais evidente na Fig. 8 onde a intensidade dos lóbulos inferiores, que dependem do campo acoplado no prisma, assim como dos lóbulos superiores, que dependem 
do campo total na primeira camada [12], aumentam de intensidade inversamente proporcional a altura do analito. Isso mostra que o modo SPP acopla-se melhor no prisma com a proximidade das nanopartículas da interface.
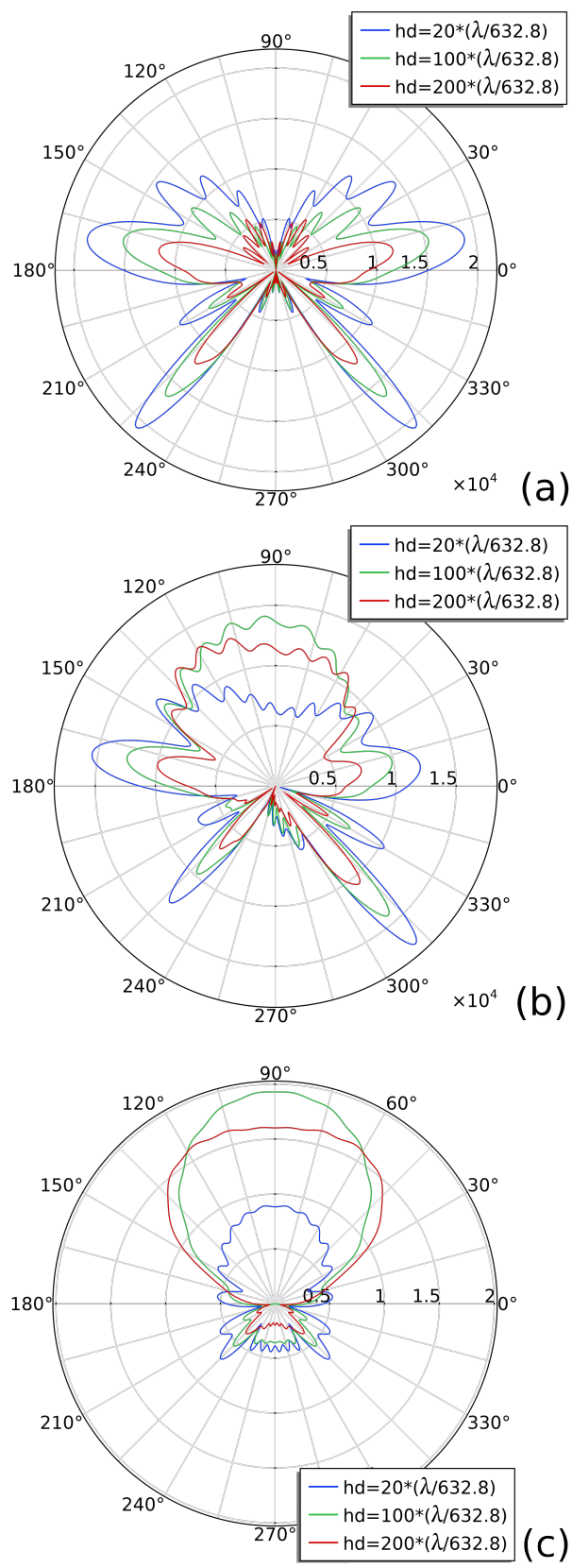

Fig. 8. Far Field $2 D$ com $h=20 \mu m, h=100 \mu m$ e $h=200 \mu m$ para $\phi^{\prime}=0^{\circ}$ e (a) $\theta^{\prime}=0^{\circ}$; (b) $\theta^{\prime}=45^{\circ}$ e (c) $\theta^{\prime}=90^{\circ}$.

\section{CONCLUSÃO}

Foi constatado que a altura do dipolo em relação a superfície do sensor (primeira camada de grafeno) afeta a intensidade de campo distante de forma inversamente proporcional, deixando os lóbulos inferiores do diagrama de radiação maiores a medida que se diminui hDipolo (Fig. 8a).

Pôde-se mostrar por meio de análise do campo elétrico e magnético para diferentes orientações do dipolo hertziano que para esse tipo de sensor a melhor configuração é com dipolo elétrico vertical (VED) resultando no acoplamento de ondas altamente polarizadas em modo TM. Na configuração de HED (Fig. 8c), há uma relativa transmissão de campo na região do prisma, mas não há excitação de SPP nas interfaces grafeno/ $/ \mathrm{SiO}_{2}$ e $\mathrm{SiO}_{2} /$ grafeno, ou seja, o campo é proveniente apenas da radiação do dipolo, não possui diretividade caracterizada pelo acoplamento de ondas plasmônicas confirmando a teoria esplanada em [4], [11].

Todos os resultados obtidos apresentam coerência e consistência, apresentando o que se espera para esse tipo de sensor: uma boa sensibilidade quando trata-se da detecção de analitos (podendo ser dentre outros, analitos químicos e biológicos) e boa diretividade de ondas TM na região do prisma que serão recebidas pelo detector.

\section{REFERÊNCIAS}

[1] K. S. Novoselov, D. Jiang, F. Schedin, T. Booth, V. Khotkevich, S. Morozov, and A. K. Geim, "Two-dimensional atomic crystals," Proceedings of the National Academy of Sciences, vol. 102, no. 30, pp. 10451-10453, 2005.

[2] F. Xia, H. Wang, D. Xiao, M. Dubey, and A. Ramasubramaniam, "Twodimensional material nanophotonics," Nature Photonics, vol. 8, no. 12, pp. 899-907, 2014.

[3] K. S. Novoselov, A. K. Geim, S. V. Morozov, D. Jiang, Y. Zhang, S. V. Dubonos, I. V. Grigorieva, and A. A. Firsov, "Electric field effect in atomically thin carbon films," Science, vol. 306, no. 5696, pp. 666-669, 2004.

[4] Y. G. da Conceição, "Análise Numérica de Sensor SPR na Configuração SPCE Baseado em Grafeno de Duas Camadas Pelo Método dos Elementos Finitos," Master's thesis, Universidade Federal do Pará, Instituto de Tecnologia, Pós-Graduação em Engenharia Elétrica, Belém, 2021.

[5] N. W. P. de Souza, J. S. Costa, R. Santos, A. F. S. da Cruz, T. Rosso, and K. Q. da Costa, Modal analysis of surface plasmon resonance sensor coupled to periodic array of core-shell metallic nanoparticles. InTech, 2017.

[6] Y. G. Conceição, A. F. S. Cruz, P. R. Amaral, P. C. Lopes, T. Del Rosso, V. Dmitriev, and K. Q. Costa, "Numerical analysis of a graphene-based spr sensor by the finite element method," in 2019 SBMO/IEEE MTT$S$ International Microwave and Optoelectronics Conference (IMOC), pp. 1-3, IEEE, 2019

[7] C. Multiphysics, "Introduction to comsol multiphysics®," COMSOL Multiphysics, Burlington, MA, accessed Feb, vol. 9, p. 2018, 1998.

[8] P. A. D. Gonçalves and N. M. Peres, An introduction to graphene plasmonics. World Scientific, 2016.

[9] W. Liu, J. Zhang, Z. Zhu, X. Yuan, and S. Qin, "Electrically tunable absorption enhancement with spectral and polarization selectivity through graphene plasmonic light trapping," Nanomaterials, vol. 6, no. 9, p. 155, 2016.

[10] A. V. Pires, "Espalhamento eletromagnético no grafeno através de transformadas de impedância," Master's thesis, Universidade Federal do Pará, Instituto de Tecnologia, Pós-Graduação em Engenharia Elétrica, Belém, 2019.

[11] A. F. S. da Cruz, "Análise de sensor plasmônico na configuração emissão acoplada via função de green 3d," Master's thesis, Universidade Federal do Pará, Instituto de Tecnologia, Pós-Graduação em Engenharia Elétrica, Belém, 2018.

[12] N. Souza, Análise de Sensores Plasmônicos Baseados em Nano Estruturas Estratificadas Utilizando o Método das Imagens Complexas Discretas [Doctoral thesis]. PhD thesis, Universidade Federal do Pará, Instituto de Tecnologia, Programa de Pós-Graduação em Engenharia Elétrica, Belém, 2018. 